\title{
The Effect of Ginger on Preventing Post-spinal Puncture Headache in Patients Undergoing Cesarean Section
}

\author{
Banafsheh Mashak $^{\circledR}$, Maryam Hashemnejad ${ }^{2}$, Kourosh Kabir ${ }^{3}$, Mansoureh Refaei ${ }^{4}$, Sara Esmaelzadeh \\ Saeieh $^{5,6}$, Shoukofeh Torkashvand ${ }^{7}$, Leili Salehi ${ }^{8}$, Mansoureh Yazdkhasti ${ }^{5,6^{*}}$
}

\begin{abstract}
Objectives: Post-spinal puncture headache (PSPH) has constantly been one of the research priorities, especially in women undergoing cesarean section (C-section) and it is related to physical and psychological problems. The present study aimed to evaluate the effect of ginger on preventing PSPH in patients undergoing C-section.

Materials and Methods: This clinical trial was conducted on 160 women undergoing C-section with spinal anesthesia, who were eligible to enter the study in the experimental and control groups. One ginger capsule ( $250 \mathrm{mg}$ ) was prescribed every 8 hours (TDS) to the experimental group 24 hours before the C-section. The prescription of ginger was continued half an hour after transfer to the post-partum ward until the PSPH onset. PSPH intensity was measured by using visual analog scale (VAS) pain scores at 6 timepoints (Time $1=30$, Time $2=60$, and Time $3=90$ minutes vs. Time $4=3$, Time $5=6$, and Time $6=12$ hours) after C-section. No interventions were performed in the control group. The data were analyzed using the SPSS software by descriptive statistics and analytical tests were applied to determine the changes in PSPH intensity.

Results: The comparison results of the mean score of PSPH intensity in the experimental and control groups indicated significant differences over time $(P<0.05)$, except for the sixth time point (12 hours after $\mathrm{C}$-section). In addition, the trend of changes at 6 timepoints based on the results of the repeated-measures test demonstrated that PSPH intensity significantly differed in the two groups over time $(P<0.001)$.

Conclusions: Based on the result, the oral prescription of ginger to women undergoing $\mathrm{C}$-section under spinal anesthesia led to effective PSPH prevention at 5 time-points (30, 60, and 90 minutes, along with 3 and 6 hours). Further, the trend of the changes represented that the intensity of PSPH decreased in the experimental group over time. Therefore, ginger is suggested as a noninvasive and efficient method used for preventing PSPH.

Keywords: Post-spinal Puncture Headache, Ginger, Cesarean Section, Prevention
\end{abstract}

\section{Introduction}

Spinal anesthesia or block is considered a form of regional anesthesia and a type of neuraxial block which involves the injection of opioids, local anesthetics, or another permissive drug into the subarachnoid space for diagnostic purposes in the neurologic sciences $(1,2)$. In addition, it is applied for providing spinal anesthesia under obstetrical and non-obstetrical conditions by injecting anesthetic agents into the subarachnoid space (3). The regional anesthesia techniques are extensively utilized for cesarean section (C-section) since they provide an overall safety with maternal and fetal profiles (4). However, nearly $40 \%$ of the cases lead to a postspinal puncture headache (PSPH) which is the most frequent complication associated with spinal a puncture. Its frequency is estimated between $1 \%$ and $70 \%$ in females depending on their age, body mass index, patient characteristics, a history of chronic headaches, headache definition, pregnancy, spinal puncture type, needle gauge, multiple dural punctures, inexpert operators, and followup methods $(2,3)$. PSPH is known by various names like post-dural puncture headache, post-lumbar puncture headache, lumbar puncture headache, post-spinal headache, and spinal headache. The risk factors for PSPH development in the related patients mainly involve females at young ages of 20-40 years with a headache history (5). Approximately $66 \%-90 \%$ of the PSPH cases occur within the first 48 and 72 hours, respectively (6). Further, the most common form of PSPH occurs in the frontal and occipital regions related to the pain diffusion into the neck and shoulders. These symptoms are due to the loss of cerebrospinal fluid and its diminished pressure, causing 
stretching and pressure on the intracerebral organs and consequent vasodilatation (7).

PSPH prophylactic measures include oral analgesia, bed rest, oral or intravenous hydration and caffeine, epidural crystalloid administration before catheter removal, and a prophylactic epidural blood patch (EBP) (8). EBP is viewed as an accepted treatment for severe PSPH. However, it is an invasive procedure associated with additional risks including another accidental dural puncture $(6,9)$.

Narcotics are used to prevent or treat PSPH; however, there is a tendency to utilize alternative medicine or other effective drugs with fewer side effects due to the undesirable side effects of narcotics or non-steroidal anti-inflammatory drugs (NSAIDs). Considering pain mitigation, ginger is possibly regarded as a suitable drug when turning to alternative medicine (10). Furthermore, it is described as an analgesic, anti-inflammatory, antipyretic, anti-spasmodic, and peripheral circulation stimulator agent (11). Additionally, the ginger plant contains various substances including carbohydrates, free fatty acids, amino acids, and compounds such as Zingiber officinale and Shagaol. In addition, gingerol and gingeridone are its two components which are considered as potent prostaglandin inhibitors with analgesic and anti-inflammatory properties. These effects are due to inhibiting cyclooxygenase (COX-2) and lipoxygenase and consequent diminishing of leukotriene and prostaglandin levels (12).

Ginger has a potential for alleviating acute pains such as menstrual (13) and post-delivery pains, as well as chronic pains and symptoms like diarrhea, vomiting, fatigue, migraine headaches (MHs), muscular and osteoarthritis pains $(14,15) . \mathrm{MH}$ is triggered by a neurovascular phenomenon, causing the stimulation of trigeminal afferent nerves and the changes in the permeability of the blood-brain barrier (16). Further, Ginger can be a good substitute for NSAIDs since it prevents neurologicalvascular disorders like migraine pains $(15,16)$.

After anesthesia, various complications including PSPH, back pain, nausea, and vomiting are observed, especially in young pregnant women in C-section, who may develop both physical and psychological problems such as increased number of hospitalization days, impaired self-care and baby-care, enhanced hospitalization costs, delayed release, mother-baby communication, and breastfeeding (17). However, no side effects or drug interactions are reported (14) for anesthesia according to Commission E Monographs (German Pharmacopeia). Nevertheless, there is insufficient evidence on the effect of ginger for preventing PSPH. Thus, the current study sought to determine its effect on PSPH prevention in patients who underwent cesarean under spinal anesthesia.

\section{Materials and Methods}

The population of this clinical trial study included eligible pregnant women undergoing a planned cesarean section
(C-section) under spinal anesthesia. The inclusion criteria were being within the age range of 20-40-year-old, having full-term and singleton pregnancies, undergoing spinal anesthesia due to the non-emergency reasons of C-section, suffering from ASA I and II (American society of anesthesiologists physical status classification), lacking a history of psychiatric problems, convulsion or current convulsion, migraine, current migraine, or recurring headaches for any reasons, as well as lacking hypothyroidism, pre-eclampsia or eclampsia, and meningitis during the study. The exclusion criteria included having a fever or unstable vital signs, as well as a history of taking psychedelic or narcotic drugs, gallstones, being addicted to alcohol or drug abuse, and demonstrating sensitivity to ginger. The present study was implemented in the Gynecology Department of Kamali Referral hospital in Karaj, Iran during (May) 2016- (February) 2018. A 3-part questionnaire was used to collect the data regarding demographic characteristics such as age, body mass index (BMI), education level, and socioeconomic status; mean arterial pressure, heart rate, arterial oxygen saturation, cesarean duration; visual analog scale (VAS) for measuring the intensity of PSPH, namely, no to mild (0-3), medium (4-7), and severe (8-10). Melzak introduced the numerical pain rating scale as a valid and reliable measure for assessing the pain intensity (18). In the pilot study, PSPH intensity was separately measured in 10 patients by both the researcher and research assistant using the above-mentioned scales while the correlation between their measurements was considered as $r=0.83$.

Furthermore, the sample size was determined to include 160 (80 patients in each group) based on the information provided by similar studies and according to the formula of sample volume calculation to find the differences of 2 means with $\alpha=5 \%, \beta=20 \%$, and power $=80 \%$ considering $10 \%$ of loss to follow up. Sampling was conducted by a simple random sampling technique. First, the eligible women, among those pregnant women, who were waiting for a planned C-section, were entered into the study after being registered in a separate list. Then, they were divided into experimental and control groups using a simple random method. Randomization opaque envelopes were pre-determinately made by a computer to seal the randomization numbers. Next, the group names of the pregnant women and their sequence-based screening of numbers were printed inside and outside of the envelopes, respectively. All the envelopes included consecutively connected numbers. The eligible patients were assigned to either the experimental or control group after being screened for pregnancy by the researchers who separated the envelopes from the strains according to the baseline and opened them based on their sequence numbers.

In this study, the effect of the ginger capsule containing $250 \mathrm{mg}$ of the powdered granule of ginger rhizome (i.e., gingerol and shogaol) was assessed on PSPH prevention. Additionally, 24 hours before of C- section, one ginger 
capsule $(250 \mathrm{mg}$ ) was prescribed to the experimental group every 8 hours (TDS). The prescription of the ginger capsule was continued half an hour after the transfer to the post-partum ward until the PSPH onset. Upon the onset of PSPH with a score of 3 based on VAS, the prescription of ginger was discontinued. Then, the routine PSPH treatment was administrated. In addition, PSPH intensity was estimated using VAS pain scores at 6 timepoints (Time $1=30$, Time $2=60$, and Time $3=90$ minutes, as well as Time $4=3$, Time $5=6$, and Time $6=12$ hours) after $\mathrm{C}$-section in the post-partum ward. No interventions were conducted in the control group.

All the patients once underwent spinal anesthesia in a sitting position by an anesthesiologist. The operation room temperature was kept at $24^{\circ} \mathrm{C}$ and the intravenous fluids received by all the patients were as much as $500 \mathrm{cc}$ in Ringer's serum 45 minutes before the spinal anesthesia and 2.5-3 L in Ringer's serum immediately after the spinal anesthesia having the same temperatures as that of the operation room. Further, the spinal anesthesia technique was performed by injecting $3 \mathrm{cc}$ of hyperbaric $0.5 \%$ bupivacaine into the intervertebral space of L3-L4 or L4-L5 with a 25-gauge Quincke needle under sterilized conditions while the anesthesia level was kept at T4-T6 level by the pinprick test. The spinal needle was introduced in the midline and straightforward. No other medication was applied in the premedication stage.

Data Analysis and Description

The data were analyzed using the SPSS software, version
20 by descriptive statistics (i.e., mean, standard deviation, and percentage) and analytical tests (i.e., chi-square, independent $t$-test, and repeated measures analysis of variances [ANOVA]). Repeated measures analysis of variances was used to determine the changes in PSPH intensity. After exclusion, eligible patients were analyzed. The related results are presented in the following CONSORT (CONsolidated Standards of Reporting Trials) flow diagram (Figure 1). A $P<0.05$ was considered as the level of significance.

\section{Results}

Totally, 160 patients were analyzed. The independent $t$ test was used for the statistical analyses of age and BMI. Furthermore, the Chi-square test was employed to analyze the education level and socioeconomic status of the patients. The demographic characteristics of the two groups demonstrated no significant differences (Table 1).

The results of the independent $t$ test indicated that none of the groups had statistically significant differences in terms of the mean arterial pressure, heart rate, arterial oxygen saturation, and cesarean duration (Table 2).

The comparison results related to the mean score of $\mathrm{PSPH}$ intensity in the experimental and control groups represented significant differences over time except for the 6th time point (12 hours after the C-section), the details of which are provided in Table 3.

Additionally, the intervention effects on changes in PSPH intensity were compared between the two groups at 6 points of time (Time $1=30$, Time $2=60$, and Time

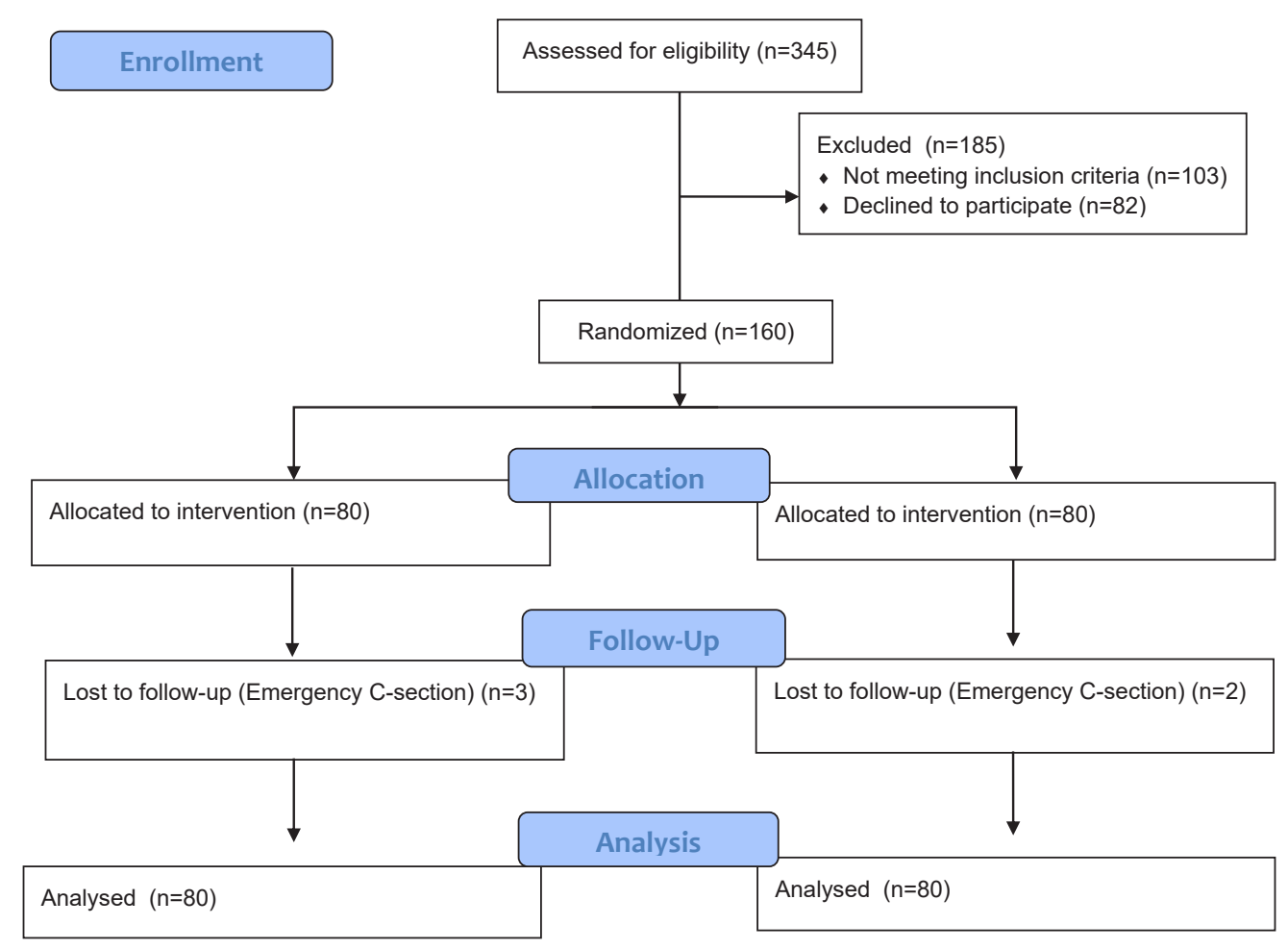

Figure 1. The Consort Flowchart. 
Table 1. The Demographic Characteristics of the 2 Groups

\begin{tabular}{lcc}
\hline Variable & Experimental $(\mathrm{n}=\mathbf{8 0})$ & Control $(\mathrm{n}=\mathbf{8 0})$ \\
\hline $\mathrm{Age}^{\mathrm{a}}(\mathrm{y})$, Mean \pm SD & $31.18 \pm 4.73$ & $29.80 \pm 6.35$ \\
$\mathrm{BMI}^{\mathrm{a}},\left(\mathrm{kg} / \mathrm{m}^{2}\right)$, mean \pm SD & $31.37 \pm 8.69$ & $29.94 \pm 4.25$ \\
Education level $^{\mathrm{b}}$, No. $(\%)$ & & 0.122 \\
Primary school & $12(15.1)$ & $5(6.3)$ \\
High school & $(23.8)$ & $20(25)$ \\
High school diploma & $41(51)$ & $51(63.6)$ \\
Advanced diploma \& bachelor's degree & $8(10.1)$ & $4(5.1)$ \\
Socioeconomic Status ${ }^{b}$, No. (\%) & & $5(6.3)$ \\
Poor & $1(1.3)$ & $67(83.8)$ \\
Moderate & $71(88.8)$ & $8(10)$ \\
Good & $8(10)$ & 0.249 \\
\hline
\end{tabular}

BMI, body mass index; SD, standard deviation.

Note. ${ }^{\mathrm{a}} \mathrm{t}$ test; ${ }^{\mathrm{b}} \mathrm{Chi}$-square test.

Table 2. The Mean Arterial Pressure, Heart Rate, Arterial Oxygen Saturation, and Cesarean Duration of Two Groups

\begin{tabular}{lcc}
\hline Parameters $^{\text {a }}$ & $\begin{array}{c}\text { Experimental }(\mathbf{n}=\mathbf{8 0}) \\
\text { Mean } \pm \text { SD }\end{array}$ & $\begin{array}{c}\text { Control ( } \mathbf{n}=\mathbf{8 0}) \\
\text { Mean } \pm \text { SD }\end{array}$ \\
\hline Mean arterial pressure (mm Hg) & $99.22 \pm 14.22$ & $98.77 \pm 12.93$ \\
Heart rate (bpm) & $98.47 \pm 15.90$ & $96.97 \pm 14.60$ \\
Arterial oxygen saturation (\%) & $98.63 \pm 1.22$ & $98.45 \pm 1.21$ \\
Cesarean duration (min) & $30.07 \pm 4.30$ & $32.53 \pm 2.06$ \\
\hline
\end{tabular}

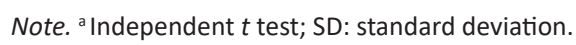

Table 3. The Comparison Results of Mean PSPH Intensity in the Experimental and Control Groups

\begin{tabular}{|c|c|c|c|c|c|}
\hline Data Point ${ }^{a}$ & $\begin{array}{c}\text { Experimental }(n=80) \\
\text { Mean } \pm S D\end{array}$ & $\begin{array}{c}\text { Control }(n=80) \\
\text { Mean } \pm S D\end{array}$ & Mean Difference & $95 \% \mathrm{Cl}$ & $P$ Value \\
\hline Time 1 & $0.14 \pm 0.52$ & $1.51 \pm 2.63$ & 1.37 & $(0.78-1.97)$ & 0.001 \\
\hline Time 2 & $0.39 \pm 0.68$ & $2.18 \pm 2.375$ & 1.78 & $(1.24-2.34)$ & 0.001 \\
\hline Time 3 & $0.29 \pm 0.70$ & $2.00 \pm 2.22$ & 1.71 & $(1.196-2.23)$ & 0.001 \\
\hline Time 4 & $0.09 \pm 0.33$ & $0.94 \pm 1.694$ & 0.85 & $(0.47-1.23)$ & 0.001 \\
\hline Time 5 & $0.04 \pm 0.23$ & $0.25 \pm 0.834$ & 0.21 & $(0.19-0.41)$ & 0.032 \\
\hline Time 6 & $0.04 \pm 0.25$ & $0.14 \pm 0.52$ & 0.10 & $(-0.28-(+0.21)$ & 0.124 \\
\hline
\end{tabular}

Note. ${ }^{\text {a }}$ Independent $t$ test; PSPH: Post-spinal puncture headache; SD: standard deviation.

$3=90$ minutes; Time $4=3$, Time $5=6$, and Time $6=12$ hours) by the repeated-measures ANOVA and the general linear model. The results of the Mauchly's test revealed that the sphericity assumption was not established $(P<$ 0.001). Therefore, Greenhouse-Geisser assumption was used to correct the degrees of freedom of the repeatedmeasures test. The results indicated that PSPH intensity significantly varied in both groups over time (Tables 4 and Figure 2).

\section{Discussion}

PSPH has been permanently considered one of the research priorities, especially in women undergoing C-section (5). In this study, a ginger capsule was employed aiming at preventing $\mathrm{PSPH}$ development. The results indicated that the mean scores PSPH were significantly different in the experimental and control groups. In addition, based on the results, ginger was effective in preventing
PSPH over time and diminished the intensity of PSPH at various time points. Comparing the results of mean score PSPH intensity between experimental and control groups revealed significant differences over time $(P<0.05)$ except for the 6th time point (i.e., 12 hours after the $\mathrm{C}$-section). The results of the repeated-measures test regarding the trend of changes at 6 points in time represented a significant difference in PSPH intensity between the two groups over time $(P<0.001)$.

The narcotics are used to prevent PSPH. Abboud et al investigated the preventive effect of morphine on the prevalence of PSPH intensity. Their double-blind clinical trial was conducted on 82 subjects undergoing C-section with spinal anesthesia. In the intervention group, 0.2 mg of morphine was applied while in the control group, saline $(0.2 \mathrm{~mL})$ mixed with $0.75 \%$ bupivacaine in $8.25 \%$ dextrose plus 1:1000 subarachnoid epinephrine (0.2 $\mathrm{mL}$ ) was analyzed before the spinal anesthesia. PSPH 
Table 4. The Analysis of Variance of PSPH at 6 Points of Time in Experimental and Control Groups

\begin{tabular}{|c|c|c|c|c|}
\hline \multirow[t]{2}{*}{ Data Point } & \multirow{2}{*}{$\begin{array}{l}\text { Experimental }(n=80) \\
\text { Mean } \pm S D\end{array}$} & \multirow{2}{*}{$\begin{array}{c}\text { Control }(n=80) \\
\text { Mean } \pm S D\end{array}$} & \multicolumn{2}{|c|}{$\begin{array}{c}\text { Repeated-Measure Test } \\
\text { Time*Group }\end{array}$} \\
\hline & & & Within Group & Between Group \\
\hline Time 1 & $0.14 \pm 0.52$ & $1.51 \pm 2.63$ & \multirow{5}{*}{$\begin{array}{c}P=0.001 \\
F=28.07 \\
S S=81.77 \\
M S=81.77\end{array}$} & \multirow{3}{*}{$\begin{array}{l}P=0.001 \\
F=42.15\end{array}$} \\
\hline Time 2 & $0.39 \pm 0.68$ & $2.18 \pm 2.375$ & & \\
\hline Time 3 & $0.29 \pm 0.70$ & $2.00 \pm 2.22$ & & \\
\hline Time 4 & $0.09 \pm 0.33$ & $0.94 \pm 1.694$ & & SS $=243.01$ \\
\hline Time 5 & $0.04 \pm 0.23$ & $0.25 \pm 0.834$ & & $M S=243.01$ \\
\hline Time 6 & $0.04 \pm 0.25$ & $0.14 \pm 0.52$ & & \\
\hline
\end{tabular}

Note. SS, Sum of square; MS, Mean square; SD: Standard deviation; PSPH: Post-spinal puncture headache.

intensity was measured for 3 days and its prevalence was tested for 2 weeks. However, the results revealed no significant differences between the two groups based on PSPH intensity and incidence at the mentioned times (19). Conversely, Martlew reported that a total of 5173 patients received spinal anesthesia for C-section during the audit period, of whom 4559 subjects were followed up, postoperatively. The results suggested that intrathecal diamorphine instead of fentanyl reduced the PSPH incidence. However, the findings of another study demonstrated that the risk failed to reduce by intrathecal fentanyl when using a 25-G Quincke needle. Eldor et al presented other reports respecting the effect of epidural morphine on headache prevention after accidental dural puncture $(21,22)$. Nevertheless, insufficient evidence exists on the preventive effects of intrathecal diamorphine on the extent of PSPH incidence and intensity (20).

The EBP is employed for PSPH prevention as well. In a systematic review and meta-analysis, Bradbury et al indicated that there was insufficient evidence for the prophylactic use of EBP for mitigating the incidence and intensity of PSPH. They highlighted several side effects for the prophylactic use of EBP including backache and substance deposition which provide a potential medium for the bacterial growth. Finally, they concluded that the usefulness of the prophylactic use of EBP cannot be confirmed without further investigations (8).

$\mathrm{MH}$ has somehow the same mechanism as headaches

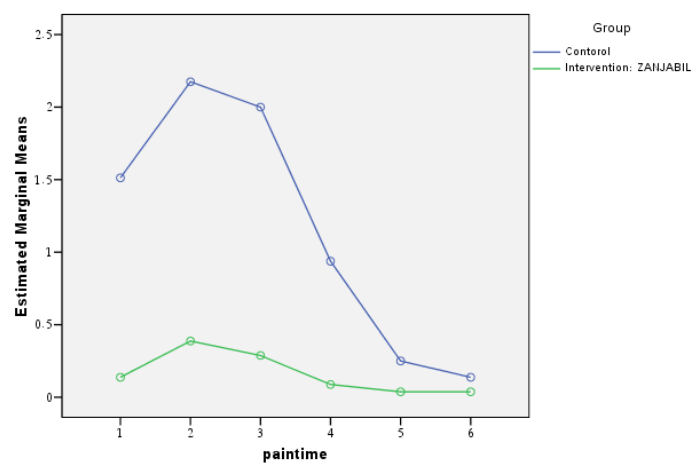

Figure 2. Visual analog Scales in Both Groups in 6 Points of Time Based on Repeated-Measures ANOVA. resulting from PSPH. The reason for this similarity is that $\mathrm{MH}$ is affected by the neurovascular phenomenon leading to the stimulation of afferent trigeminal nerve and the variations of blood-brain barrier permeability (16). In a double-blind clinical trial, Maghbooli et al compared the effects of ginger and sumatriptan on patients with acute $\mathrm{MH}$ without aura. The severity of headache was measured by the VAS at 6 time-points (before the intervention and at the times of $30,60,90$, and 120 minutes and 24 hours). The results demonstrated the similar effects of ginger and sumatriptan on the time of headache onset and its intensity. Further, fewer side effects were reported for the ginger group of patients (15). Furthermore, Cady et al enrolled 30 patients involved in the mild pain phase of GelStat ${ }^{\circledR}$ Migraine and attempted to treat them with a combination of ginger and feverfew. After 2 hours of treatment, the subjects still had the same feeling as what they felt before the medication (41\%), reported recurrence of their headaches within 24 hours with only minimal side effects (29\%), reported only mild headaches (34\%), were pain-free (48\%), and satisfied (59\%), respectively. In the present study, 2 hours after ginger intake, the patients were involved in clinical adverse reactions (4\%) or were painfree $(56 \%)$. Additionally, $88 \%$ of the patients reported only mild headaches while $44 \%$ of them reported excellent or great headache reliefs. Finally, nearly $72 \%$ of the patients satisfactorily preferred our drug for their long-term therapies (23).

In addition, glucocorticoid products were utilized to prevent $\mathrm{PSPH}$, due to their preventive or antiinflammatory effects. These products prevent or treat $\mathrm{PSPH}$ they inhibit pain-sensitive vasodilatation inside the brain. Moral et al applied the intravenous injection of hydrocortisone to prevent PSPH. This intervention was effective and thus, was recommended as an alternative approach to EBP (24). Further, Colman et al assessed intravenous dexamethasone for treating recurrent $\mathrm{MH}$ and found that it was considerably effective in mitigating the recurrence of $\mathrm{MH}$ (25). Although the mechanisms of ginger effect have not been completely investigated, it may lead to diminished vasodilatation inside the brain and PSPH intensity possibly due to the neurogenic anti- 
inflammatory effects (15). Similarly, based on the results of the current study, ginger decreased PSPH intensity at various time points in the experimental group.

Furthermore, ginger demonstrates positive effects on dysmenorrhea by other mechanisms. It relieves dysmenorrhea in various ways. The effect of ginger on dysmenorrhea is dependent on the inhibitions of thromboxane and prostaglandin activities (13). Prostaglandins are produced by arachidonic acid cyclooxygenase and lipoxygenase (26). There are higher concentrations of prostaglandin F2 $\alpha$ and E2 in the menstruation blood of women suffering from dysmenorrhea (27). Additionally, NSAIDs such as mefenamic acid prevent prostaglandin synthesis by inhibiting the COX-2 activity (28).

In addition, Halder et al compared the effects of ginger powder, ginger tablet, and placebo on the treatment of primary dysmenorrhea. The results revealed that ginger in its both powder and tablet forms caused improved menstrual pains (29). In another study, Shirvani et al (13) compared the effects of ginger and mefenamic acid on the intensity of primary dysmenorrhea. To this end, ginger $(250 \mathrm{mg}$ ) and 250-mg mefenamic acid capsules were prescribed once TDS from the beginning of the menstruation cycle. The results revealed that ginger and mefenamic acid had similar mitigatory effects on the intensity of primary dysmenorrhea at 3 time-points (onset, 1st, and 3rd month). Further, Ozgoli et al found that ginger had the same effects as mefenamic acid and ibuprofen on mitigating the primary intensity of dysmenorrhea after prescribing as a $250 \mathrm{~g}$-capsule once every 6 hours (30). Similarly, Rahnama et al reported significantly lower duration of pain when prescribing ginger 2 days before the onset until 3 days after the menstruation compared to the time it was taken at the menstruation onset. Thus, they concluded that the early start of ginger intake possibly reduces the pain severity (10).

Furthermore, in the current study, ginger was prescribed to prevent PSPH at a dose of $250 \mathrm{mg}$ TDS before spinal anesthesia. The ginger capsule was confirmed to diminish the intensity of PSPH in the experimental group during several time-points.

Ginger is further found to be a suitable drug for alleviating the rheumatic pains since it has anti-inflammatory effects. In the Ayurvedic and Tibbi systems of medicine, ginger is described as a useful anti-inflammatory and antirheumatic drug (11). Therefore, these effects of ginger led to alleviated PSPH intensity in the experimental group of the present study.

\section{Conclusions}

Based on the above-mentioned studies, there are still no standard approach for preventing the PSPH. In general, the result demonstrated that the oral prescription of ginger to women undergoing C-section under spinal anesthesia leads to effective PSPH prevention at 5 timepoints (30,60, and 90 minutes, along with 3 and 6 hours). Additionally, the trend of the changes indicated that the intensity of PSPH decreased in the experimental group over time. Therefore, ginger is recommended as a noninvasive and efficient method for preventing PSPH.

\section{Limitations of the Study and Solutions}

In every human attempt, no doubt, there exist some limitations which need to be acknowledged.

No study was found to deal with the preventive or therapeutic effects of ginger on $\mathrm{PSPH}$ in order to compare its findings with the results of the present study. Thus, other similar studies were reviewed, which dealt with its preventive or therapeutic effects on MHs, dysmenorrhea, and osteoarthritis. In addition, in the reviewed studies, ginger was used at different doses to pursue varied therapeutic goals. Further, in the self-report of PSPH measurements through VAS, education level and socioeconomic status probably acted as confounding variables and thus were resolved by homogenizing the involved groups by randomization.

\section{Conflict of Interests}

Authors declare that they have no conflict of interests.

\section{Ethical Issues}

This study was approved by the Medical Research and Ethical Committee of Alborz University of Medical Sciences (decree number $=$ Abzums.rec.1396.7; IRCT number: IRCT20180110038302N1). All the participants signed a consent form, in which the study procedures were explained.

\section{Financial Support}

This research was supported by Alborz University of Medical Sciences.

\section{Acknowledgments}

We highly appreciate the Vice-president for the research at Alborz University of Medical Sciences for his support, as well as the study participants and the personnel of Post-partum Ward, Department of Gynecology, Kamali Referral Hospital, who kindly assisted us in conducting this research.

\section{References}

1. Apfel CC, Saxena A, Cakmakkaya OS, Gaiser R, George E, Radke O. Prevention of postdural puncture headache after accidental dural puncture: a quantitative systematic review. Br J Anaesth. 2010;105(3):255-263. doi:10.1093/bja/aeq191

2. Basurto Ona X, Martinez Garcia L, Sola I, Bonfill Cosp X. Drug therapy for treating post-dural puncture headache. Cochrane Database Syst Rev. 2011(8):Cd007887. doi:10.1002/14651858.CD007887.pub2

3. Halker RB, Demaerschalk BM, Wellik KE, et al. 
Caffeine for the prevention and treatment of postdural puncture headache: debunking the myth. Neurologist. 2007;13(5):323-327. doi:10.1097/NRL.0b013e318145480f

4. Tsen LC, Boosalis P, Segal S, Datta S, Bader AM. Hemodynamic effects of simultaneous administration of intravenous ephedrine and spinal anesthesia for cesarean delivery. J Clin Anesth. 2000;12(5):378-382.

5. Flood P. Postdural puncture headache in obstetrics. Semin Perinatol. 2002;26(2):146-153.

6. Mason L, Edwards JE, Moore RA, McQuay HJ. Single-dose oral naproxen for acute postoperative pain: a quantitative systematic review. BMC Anesthesiol. 2003;3(1):4. doi:10.1186/1471-2253-3-4

7. Usta B, Gozdemir M, Demircioglu RI, Muslu B, Sert $\mathrm{H}$, Yaldiz A. Dexmedetomidine for the prevention of shivering during spinal anesthesia. Clinics (Sao Paulo). 2011;66(7):1187-1191.

8. Bradbury CL, Singh SI, Badder SR, Wakely LJ, Jones PM. Prevention of post-dural puncture headache in parturients: a systematic review and meta-analysis. Acta Anaesthesiol Scand. 2013;57(4):417-430. doi:10.1111/aas.12047

9. Banks S, Paech M, Gurrin L. An audit of epidural blood patch after accidental dural puncture with a Tuohy needle in obstetric patients. Int J Obstet Anesth. 2001;10(3):172176. doi:10.1054/ijoa.2000.0826

10. Rahnama P, Montazeri A, Huseini HF, Kianbakht S, Naseri M. Effect of Zingiber officinale R. rhizomes (ginger) on pain relief in primary dysmenorrhea: a placebo randomized trial. BMC Complement Altern Med. 2012;12:92. doi:10.1186/1472-6882-12-92

11. Srivastava KC, Mustafa T. Ginger (Zingiber officinale) in rheumatism and musculoskeletal disorders. Med Hypotheses. 1992;39(4):342-348. doi:10.1016/03069877(92)90059-L

12. Chrubasik S, Pittler MH, Roufogalis BD. Zingiberis rhizoma: a comprehensive review on the ginger effect and efficacy profiles. Phytomedicine. 2005;12(9):684-701. doi:10.1016/j.phymed.2004.07.009

13. Shirvani MA, Motahari-Tabari N, Alipour A. The effect of mefenamic acid and ginger on pain relief in primary dysmenorrhea: a randomized clinical trial. Arch Gynecol Obstet. 2015;291(6):1277-1281. doi:10.1007/s00404-0143548-2

14. Ding M, Leach MJ, Bradley H. A systematic review of the evidence for topical use of ginger. Explore (NY). 2013;9(6):361-364. doi:10.1016/j.explore.2013.08.001

15. Maghbooli M, Golipour F, Moghimi Esfandabadi A, Yousefi M. Comparison between the efficacy of ginger and sumatriptan in the ablative treatment of the common migraine. Phytother Res. 2014;28(3):412-415. doi:10.1002/ ptr.4996

16. Goadsby PJ. Recent advances in understanding migraine mechanisms, molecules and therapeutics. Trends Mol Med. 2007;13(1):39-44. doi:10.1016/j.molmed.2006.11.005

17. Leach MJ. Profile of the complementary and alternative medicine workforce across Australia, New Zealand, Canada, United States and United Kingdom. Complement Ther Med. 2013;21(4):364-378. doi:10.1016/j.ctim.2013.04.004

18. Sindhu F. Are non-pharmacological nursing interventions for the management of pain effective?--A meta-analysis. J Adv Nurs. 1996;24(6):1152-1159.

19. Abboud TK, Zhu J, Reyes A, et al. Effect of subarachnoid morphine on the incidence of spinal headache. Reg Anesth. 1992;17(1):34-36.

20. Martlew RA. Spinal opioids and the prevention of post dural puncture headache. Anaesthesia. 2009;64(1):97. doi:10.1111/j.1365-2044.2008.05803.x

21. Eldor J, Guedj P, Cotev S. Epidural morphine injections for the treatment of postspinal headache. Can J Anaesth. 1990;37(6):710-711. doi:10.1007/bf03006510

22. Bradbury CL, Singh SI, Badder SR, Wakely LJ, Jones PM. Prevention of post-dural puncture headache in parturients: a systematic review and meta-analysis. Acta Anaesthesiol Scand. 2013;57(4):417-430. doi:10.1111/aas.12047

23. Cady RK, Schreiber CP, Beach ME, Hart CC. Gelstat Migraine (sublingually administered feverfew and ginger compound) for acute treatment of migraine when administered during the mild pain phase. Med Sci Monit. 2005;11(9):Pi65-69.

24. Moral Turiel M, Rodriguez Simon MO, Sahagun de la Lastra J, Yuste Pascual JA. [Treatment of post-dural-puncture headache with intravenous cortisone]. Rev Esp Anestesiol Reanim. 2002;49(2):101-104.

25. Colman I, Friedman BW, Brown MD, et al. Parenteral dexamethasone for acute severe migraine headache: metaanalysis of randomised controlled trials for preventing recurrence. BMJ. 2008;336(7657):1359-1361. doi:10.1136/ bmj.39566.806725.BE

26. Grzanna R, Lindmark L, Frondoza CG. Ginger--an herbal medicinal product with broad anti-inflammatory actions. J Med Food. 2005;8(2):125-132. doi:10.1089/jmf.2005.8.125

27. Bieglmayer C, Hofer G, Kainz C, Reinthaller A, Kopp B, Janisch H. Concentrations of various arachidonic acid metabolites in menstrual fluid are associated with menstrual pain and are influenced by hormonal contraceptives. Gynecol Endocrinol. 1995;9(4):307-312. doi:10.3109/09513599509160464

28. Marjoribanks J, Proctor M, Farquhar C, Derks RS. Nonsteroidal anti-inflammatory drugs for dysmenorrhoea. Cochrane Database Syst Rev. 2010(1):Cd001751. doi:10.1002/14651858.CD001751.pub2

29. Halder A. Effect of progressive muscle relaxation versus intake of ginger powder on dysmenorrhoea amongst the nursing students in Pune. Nurs J India. 2012;103(4):152156.

30. Ozgoli G, Goli M, Moattar F. Comparison of effects of ginger, mefenamic acid, and ibuprofen on pain in women with primary dysmenorrhea. J Altern Complement Med. 2009;15(2):129-132. doi:10.1089/acm.2008.0311

(C) 2019 The Author (s); This is an open-access article distributed under the terms of the Creative Commons Attribution License (http://creativecommons.org/licenses/by/4.0), which permits unrestricted use, distribution, and reproduction in any medium, provided the original work is properly cited. 